\title{
Review of: "Antibacterial efficacy of non-thermal atmospheric plasma against Streptococcus mutans biofilm grown on the surfaces of restorative resin composites"
}

\author{
Fernanda Tome
}

Potential competing interests: The author(s) declared that no potential competing interests exist.

Congratulations to the authors for the research.

Some observations on the content of the research:

1. In the methods after chlorhexidine application was washed the material?

2. On the discussion when talking about the effectiveness of the proposed treatment, being an efficacy below $50 \%$ that I suggest talking about a partial efficacy.

3. Also on the discussion it is important to mention the structure of the extracellular matrix ofS. mutans and then relate the results, including as a possible barrier to less efficacy of plasma treatment in relation to chlorhexidine.

4. Studies with longer biofilms would be interesting since the extracellular matrix and the organization of the biofilm would be larger.

5. The discussion left to be desired in the explanations on theoretical bases about the mechanisms involved in the results obtained, as well as the demonstration of other studies.

6. What would be the advantage of plasma over chlorhexidine since the plasma result is much lower than that of conventional chlorhexidine?

7. The PCR study seemed quite superficial, I believe it should be improved.

8. With regard to the many references are outdated and very old, I suggest seeking more current references. 